\title{
Estimation of Sobolev embedding constant on a domain dividable into bounded convex domains
}

\author{
Makoto Mizuguchi ${ }^{*}$, Kazuaki Tanaka', Kouta Sekine ${ }^{2}$ and Shin'ichi Oishi ${ }^{1}$
}

\section{"Correspondence:}

makoto.math@aoni.waseda.jp

${ }^{1}$ Faculty of Science and

Engineering, Waseda University,

3-4-1, Okubo Shinjyuku-ku, Tokyo 169-8555, Japan

Full list of author information is available at the end of the article

\begin{abstract}
This paper is concerned with an explicit value of the embedding constant from $W^{1, q}(\Omega)$ to $L^{p}(\Omega)$ for a domain $\Omega \subset \mathbb{R}^{N}(N \in \mathbb{N})$, where $1 \leq q \leq p \leq \infty$. We previously proposed a formula for estimating the embedding constant on bounded and unbounded Lipschitz domains by estimating the norm of Stein's extension operator. Although this formula can be applied to a domain $\Omega$ that can be divided into a finite number of Lipschitz domains, there was room for improvement in terms of accuracy. In this paper, we report that the accuracy of the embedding constant is significantly improved by restricting $\Omega$ to a domain dividable into bounded convex domains.
\end{abstract}

MSC: 46E35

Keywords: Sobolev embedding constant; Hardy-Littlewood-Sobolev inequality; Young inequality

\section{Introduction}

We consider the Sobolev type embedding constant $C_{p}(\Omega)$ from $W^{1, q}(\Omega)(1 \leq q \leq p \leq \infty)$ to $L^{p}(\Omega)$. The constant $C_{p}(\Omega)$ satisfies

$$
\left(\int_{\Omega}|u(x)|^{p} d x\right)^{\frac{1}{p}} \leq C_{p}(\Omega)\left(\int_{\Omega}|u(x)|^{q} d x+\int_{\Omega}|\nabla u(x)|^{q} d x\right)^{\frac{1}{q}}
$$

for all $u \in W^{1, q}(\Omega)$, where $\Omega \subset \mathbb{R}^{N}(N \in \mathbb{N})$ is a bounded domain and $|x|=\sqrt{\sum_{j=1}^{N} x_{j}^{2}}$ for $x=\left(x_{1}, \ldots, x_{N}\right) \in \mathbb{R}^{N}$. Here, $L^{p}(\Omega)(1 \leq p<\infty)$ is the functional space of the $p$ th power Lebesgue integrable functions over $\Omega$ endowed with the norm $\|f\|_{L^{p}(\Omega)}:=\left(\int_{\Omega}|f(x)|^{p} d x\right)^{1 / p}$ for $f \in L^{p}(\Omega)$, and $L^{\infty}(\Omega)$ is the functional space of Lebesgue measurable functions over $\Omega$

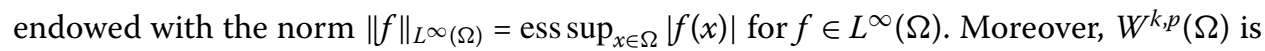
the $k$ th order $L^{p}$-Sobolev space on $\Omega$ endowed with the norm $\|f\|_{W^{1, p}(\Omega)}=\left(\int_{\Omega}|f(x)|^{p} d x+\right.$ $\left.\int_{\Omega}|\nabla f(x)|^{p} d x\right)^{1 / p}$ for $f \in W^{1, p}(\Omega)$ if $1 \leq p<\infty$ and $\|f\|_{W^{1, \infty}(\Omega)}=\operatorname{ess} \sup _{x \in \Omega}|f(x)|+$ ess $\sup _{x \in \Omega}|\nabla f(x)|$ for $f \in W^{1, \infty}(\Omega)$ if $p=\infty$.

Since inequality (1) has significance for studies on partial differential equations, many researchers studied this type of Sobolev inequality and an explicit value of $C_{p}(\Omega)$ (see, e.g., [1-7]) following the pioneering work by Sobolev [1]. In particular, our interest is in the applicability of this constant to verified numerical computation methods for PDEs

(c) The Author(s) 2017. This article is distributed under the terms of the Creative Commons Attribution 4.0 International License (http://creativecommons.org/licenses/by/4.0/), which permits unrestricted use, distribution, and reproduction in any medium, provided you give appropriate credit to the original author(s) and the source, provide a link to the Creative Commons license, and indicate if changes were made. 
which originate from Nakao's [8] and Plum's work [9]. These methods have been further developed by many researchers (see, e.g., [8-10] and the references therein).

The existence of $C_{p}(\Omega)$ for various domains $\Omega$ (e.g., domains with the cone condition, domains with the Lipschitz boundary, and the $(\varepsilon, \delta)$-domains) has been proven by constructing suitable extension operators from $W^{k, p}(\Omega)$ to $W^{k, p}\left(\mathbb{R}^{N}\right)$ (see, e.g., [3-7]).

Several formulas for computing explicit values of $C_{p}(\Omega)$ have been proposed under suitable conditions. For example, the best constant in the classical Sobolev inequality on $\mathbb{R}^{N}$ was independently shown by Aubin [11] and Talenti [12]. For the case in which $N=1$ and $p=\infty$, the best constant of $C_{p}(\Omega)$ was proposed under some boundary conditions, e.g., the Dirichlet, the Neumann, and the periodic condition [13-17]. For a square domain $\Omega \subset \mathbb{R}^{2}$, a tight estimate of $C_{p}(\Omega)$ was provided in [10]. Moreover, the best constant for the embed$\operatorname{ding} W_{0}^{1,2}(\Omega) \hookrightarrow L^{p}(\Omega)(p=3,4,5,6,7)$ with a square domain $\Omega \subset \mathbb{R}^{2}$ was very sharply estimated in [18], where $W_{0}^{1,2}(\Omega)$ denotes the closure of $C_{0}^{\infty}(\Omega)$ in $W^{1,2}(\Omega)$. Furthermore, we have previously proposed a formula for computing an explicit value of $C_{p}(\Omega)$ for (bounded and unbounded) Lipschitz domains $\Omega \subset \mathbb{R}^{N}(N \geq 2)$ by estimating the norm of Stein's extension operator [19]. This formula can be applied to a domain $\Omega$ that can be divided into a finite number of Lipschitz domains $\Omega_{i}(i=1,2,3, \ldots, n)$ such that

$$
\bar{\Omega}=\bigcup_{1 \leq i \leq n} \overline{\Omega_{i}}
$$

and

$$
\Omega_{i} \cap \Omega_{j}=\phi \quad(i \neq j),
$$

where $\phi$ is the empty set and $\bar{\Omega}$ denotes the closure of $\Omega$ (see Theorem 6.1). Although this formula is applicable to such general domains, the values computed by this formula are very large; see Section 4 for concrete values.

In this paper, we report that the accuracy of the estimation of $C_{p}(\Omega)$ is significantly improved by restricting each $\Omega_{i}$ to bounded convex domain. Since any bounded convex domain is a Lipschitz domain (see, e.g., [20]), the present class of $\Omega$ is somewhat special compared with the class treated in [19]. Nevertheless, the formulas presented in this paper still have applicability to various domains. To obtain a sharper estimation of $C_{p}(\Omega)$, we focus on the constants $D_{p}(\Omega)$ such that

$$
\left(\int_{\Omega}\left|u(x)-u_{\Omega}(x)\right|^{p} d x\right)^{\frac{1}{p}} \leq D_{p}(\Omega)\left(\int_{\Omega}|\nabla u(x)|^{q} d x\right)^{\frac{1}{q}} \quad \text { for all } u \in W^{1, q}(\Omega) .
$$

Here, $|\Omega|$ is the measure of $\Omega$ and $u_{\Omega}: \Omega \rightarrow \mathbb{R}$ is a constant function defined by $\Omega \ni x \mapsto$ $u_{\Omega}(x)=|\Omega|^{-1} \int_{\Omega} u(y) d y$. Inequality $(4)$ is called the Sobolev-Poincaré inequality, and $D_{p}(\Omega)$ in (4) leads to the explicit value of $C_{p}(\Omega)$ (see Theorem 2.1). Inequality (4) has also been studied by many researchers (see, e.g., [21-24]). For example, for a John domain $\Omega$, the existence of $D_{p}(\Omega)$ was shown while assuming that $1 \leq q<N, p=N q /(N-q)$ [23]. It was also shown that, when $p \neq N q /(N-q), D_{p}(\Omega)$ exists if and only if $W^{1, q}(\Omega)$ is continuously embedded into $L^{p}(\Omega)$ [24]. Moreover, there are several formulas for obtaining an explicit value of $D_{p}(\Omega)$ for one-dimensional domains $\Omega$ [25-27]. In the higher-dimensional cases, 
Table 1 The assumptions of $p, q$, and $N$ imposed on Theorems 3.1, 3.2, 3.3, and 3.4

\begin{tabular}{llll}
\hline Theorem & $\boldsymbol{p}$ & $\boldsymbol{q}$ & $\boldsymbol{N}$ \\
\hline 3.1 & $2<p \leq \frac{2 N}{N-1}(N>1), 2<p<\infty(N=1)$ & $q \geq \frac{p}{p-1}$ & $N \geq 1$ \\
3.2 & $2<p \leq \frac{2 N}{N-2}(N>2), 2<p<\infty(N=2)$ & $q=2$ & $N \geq 2$ \\
3.3 & $q \leq p<\frac{q N}{N-q}(N>q), q \leq p<\infty(N=q)$ & $q \geq 1$ & $N \geq q$ \\
3.4 & $p=\infty$ & $q \geq 1$ & $N<q$ \\
\hline
\end{tabular}

however, little is known about explicit values of $D_{p}(\Omega)$, except for some special cases (see, e.g., [28] and [29] for the cases in which $p=q=1$ and $p=q=2$, respectively).

We propose four theorems (Theorem 3.1 to 3.4) for obtaining explicit values of $D_{p}(\Omega)$ on a bounded convex domain $\Omega$. Each theorem can be used under the corresponding conditions listed in Table 1.

Theorems 3.1 and 3.2 are derived from the best constant in the Hardy-LittlewoodSobolev inequality on $\mathbb{R}^{N}$. Theorems 3.3 and 3.4 are derived from the best constant in Young's inequality on $\mathbb{R}^{N}$. The values of $D_{p}(\Omega)$ calculated by these theorems yield the explicit values of $C_{p}(\Omega)$ combined with Theorem 2.1.

The remainder of this paper is organized as follows. In Section 2, we propose Theorem 2.1 in which a formula for deriving an explicit value of $C_{p}(\Omega)$ from known $D_{p}(\Omega)$ is provided. In Section 3, we prove the four formulas (Theorems 3.1 to 3.4) for obtaining the explicit values of $D_{p}(\Omega)$. In Section 4 , we present examples where explicit values of $C_{p}(\Omega)$ are estimated for certain domains.

\section{Estimation of embedding constant $C_{p}(\Omega)$}

The following notation is used throughout this paper. For any bounded domain $S \subset \mathbb{R}^{N}$ $(N \in \mathbb{N})$, we define $d_{S}:=\sup _{x, y \in S}|x-y|$. The closed ball centered around $z \in \mathbb{R}^{N}$ with radius $\rho>0$ is denoted by $B(z, \rho):=\left\{x \in \mathbb{R}^{N}|| x-z \mid \leq \rho\right\}$. For $m \geq 1$, let $m^{\prime}$ be Hölder's conjugate of $m$, that is, $m^{\prime}$ is defined by

$$
\begin{cases}m^{\prime}=\infty, & \text { if } m=1, \\ m^{\prime}=\frac{m}{m-1}, & \text { if } 1<m<\infty \\ m^{\prime}=1, & \text { if } m=\infty\end{cases}
$$

For two domains $\Omega \subseteq \mathbb{R}^{N}$ and $\Omega^{\prime} \subseteq \mathbb{R}^{N}$ such that $\Omega \subseteq \Omega^{\prime}$, we define the operator $E_{\Omega, \Omega^{\prime}}$ : $L^{p}(\Omega) \rightarrow L^{p}\left(\Omega^{\prime}\right)(1 \leq p \leq \infty)$ by

$$
\left(E_{\Omega, \Omega^{\prime}} f\right)(x)= \begin{cases}f(x), & x \in \Omega, \\ 0, & x \in \Omega^{\prime} \backslash \Omega\end{cases}
$$

for $f \in L^{p}(\Omega)$. Note that $E_{\Omega, \Omega^{\prime}} f \in L^{p}\left(\Omega^{\prime}\right)$ satisfies

$$
\left\|E_{\Omega, \Omega^{\prime}} f\right\|_{L^{p}\left(\Omega^{\prime}\right)}=\|f\|_{L^{p}(\Omega)} .
$$

In the following theorem, we provide a formula for obtaining an explicit value of $C_{p}(\Omega)$ from known $D_{p}(\Omega)$. 
Theorem 2.1 Let $\Omega \subset \mathbb{R}^{N}(N \in \mathbb{N})$ be a bounded domain, and let $p$ and $q$ satisfy $1 \leq q \leq$ $p \leq \infty$. Suppose that there exists a finite number of bounded domains $\Omega_{i}(i=1,2,3, \ldots, n)$ satisfying (2) and (3). Moreover, suppose that, for every $\Omega_{i}(i=1,2,3, \ldots, n)$, there exist constants $D_{p}\left(\Omega_{i}\right)$ such that

$$
\left\|u-u_{\Omega_{i}}\right\|_{L^{p}\left(\Omega_{i}\right)} \leq D_{p}\left(\Omega_{i}\right)\|\nabla u\|_{L^{q}\left(\Omega_{i}\right)} \quad \text { for all } u \in W^{1, q}\left(\Omega_{i}\right) .
$$

Then (1) holds valid for

$$
C_{p}(\Omega)= \begin{cases}\max \left(1, \max _{1 \leq i \leq n} D_{\infty}\left(\Omega_{i}\right)\right) & (p=q=\infty), \\ 2^{1-\frac{1}{q}} \max \left(\max _{1 \leq i \leq n}\left|\Omega_{i}\right|^{\frac{1}{p}-\frac{1}{q}}, \max _{1 \leq i \leq n} D_{p}\left(\Omega_{i}\right)\right) & \text { (otherwise) }\end{cases}
$$

where this formula is understood with $1 / \infty=0$ when $p=\infty$ and/or $q=\infty$.

Proof Let $u \in W^{1, q}(\Omega)$. Since every $\Omega_{i}$ is bounded, Hölder's inequality states that

$$
\begin{aligned}
\left\|u_{\Omega_{i}}\right\|_{L^{p}\left(\Omega_{i}\right)} & =\left.\left|\int_{\Omega_{i}}\right| \Omega_{i}\right|^{-1} u(y) d y \mid\|1\|_{L^{p}\left(\Omega_{i}\right)} \\
& \leq\left|\Omega_{i}\right|^{-1+\frac{1}{q^{\prime}}}\|u\|_{L^{q}\left(\Omega_{i}\right)}\left|\Omega_{i}\right|^{\frac{1}{p}} \\
& =\left|\Omega_{i}\right|^{\frac{1}{p}-\frac{1}{q}}\|u\|_{L^{q}\left(\Omega_{i}\right)} .
\end{aligned}
$$

We describe the following proof separately for the case of $p=\infty$ and $p<\infty$.

When $p=\infty$, we have

$$
\begin{aligned}
\|u\|_{L^{\infty}(\Omega)} & =\max _{1 \leq i \leq n}\|u\|_{L^{\infty}\left(\Omega_{i}\right)} \\
& \leq \max _{1 \leq i \leq n}\left(\left\|u_{\Omega_{i}}\right\|_{L^{\infty}\left(\Omega_{i}\right)}+\left\|u-u_{\Omega_{i}}\right\|_{L^{\infty}\left(\Omega_{i}\right)}\right) .
\end{aligned}
$$

From (5) and (7), it follows that

$$
\begin{aligned}
& \|u\|_{L^{\infty}(\Omega)} \\
& \quad \leq \max _{1 \leq i \leq n}\left(\left|\Omega_{i}\right|^{-\frac{1}{q}}\|u\|_{L^{q}\left(\Omega_{i}\right)}+D_{\infty}\left(\Omega_{i}\right)\|\nabla u\|_{L^{q}\left(\Omega_{i}\right)}\right) \\
& \quad \leq \max \left\{\max _{1 \leq i \leq n}\left|\Omega_{i}\right|^{-\frac{1}{q}} \max _{1 \leq i \leq n} D_{\infty}\left(\Omega_{i}\right)\right\} \max _{1 \leq i \leq n}\left(\|u\|_{L^{q}\left(\Omega_{i}\right)}+\|\nabla u\|_{L^{q}\left(\Omega_{i}\right)}\right) .
\end{aligned}
$$

This implies that Theorem 2.1 holds for the case of $p=\infty$ and $q=\infty$.

For $q<\infty$, we have

$$
\begin{aligned}
& \|u\|_{L^{\infty}(\Omega)} \\
& \quad \leq \max \left\{\max _{1 \leq i \leq n}\left|\Omega_{i}\right|^{-\frac{1}{q}} \max _{1 \leq i \leq n} D_{\infty}\left(\Omega_{i}\right)\right\}\left(\sum_{1 \leq i \leq n}\left(\|u\|_{L^{q}\left(\Omega_{i}\right)}+\|\nabla u\|_{L^{q}\left(\Omega_{i}\right)}\right)^{q}\right)^{\frac{1}{q}} \\
& \quad \leq 2^{1-\frac{1}{q}} \max \left\{\max _{1 \leq i \leq n}\left|\Omega_{i}\right|^{-\frac{1}{q}} \max _{1 \leq i \leq n} D_{\infty}\left(\Omega_{i}\right)\right\}\|u\|_{W^{1, q}(\Omega)}
\end{aligned}
$$

where the last inequality follows from $(s+t)^{q} \leq 2^{q-1}\left(s^{q}+t^{q}\right)$ for $s, t \geq 0$. 
When $p<\infty$, we have

$$
\begin{aligned}
\|u\|_{L^{p}(\Omega)} & =\left(\sum_{1 \leq i \leq n} \int_{\Omega_{i}}|u(y)|^{p} d y\right)^{\frac{1}{p}} \\
& =\left(\sum_{1 \leq i \leq n}\|u\|_{L^{p}\left(\Omega_{i}\right)}^{p}\right)^{\frac{1}{p}} \\
& \leq\left(\sum_{1 \leq i \leq n}\left(\left\|u_{\Omega_{i}}\right\|_{L^{p}\left(\Omega_{i}\right)}+\left\|u-u_{\Omega_{i}}\right\|_{L^{p}\left(\Omega_{i}\right)}\right)^{p}\right)^{\frac{1}{p}} .
\end{aligned}
$$

From (5) and (7), it follows that

$$
\begin{aligned}
\|u\|_{L^{p}(\Omega)} & \leq\left(\sum_{1 \leq i \leq n}\left(\left|\Omega_{i}\right|^{\frac{1}{p}-\frac{1}{q}}\|u\|_{L^{q}\left(\Omega_{i}\right)}+D_{p}\left(\Omega_{i}\right)\|\nabla u\|_{L^{q}\left(\Omega_{i}\right)}\right)^{p}\right)^{\frac{1}{p}} \\
& \leq\left(\sum_{1 \leq i \leq n}\left(\left|\Omega_{i}\right|^{\frac{1}{p}-\frac{1}{q}}\|u\|_{L^{q}\left(\Omega_{i}\right)}+D_{p}\left(\Omega_{i}\right)\|\nabla u\|_{\left.L^{q}\left(\Omega_{i}\right)\right)^{q}}\right)^{\frac{1}{q}}\right. \\
& \leq 2^{1-\frac{1}{q}}\left(\sum_{1 \leq i \leq n}\left(\left|\Omega_{i}\right|^{\frac{q}{p}-1}\|u\|_{L^{q}\left(\Omega_{i}\right)}^{q}+D_{p}\left(\Omega_{i}\right)^{q}\|\nabla u\|_{L^{q}\left(\Omega_{i}\right)}^{q}\right)\right)^{\frac{1}{q}} .
\end{aligned}
$$

Therefore, we obtain

$$
\|u\|_{L^{p}(\Omega)} \leq 2^{1-\frac{1}{q}} \max \left\{\max _{1 \leq i \leq n}\left|\Omega_{i}\right|^{\frac{1}{p}-\frac{1}{q}}, \max _{1 \leq i \leq n} D_{i}\left(\Omega_{i}\right)\right\}\|u\|_{W^{1, q}(\Omega)} .
$$

\section{Estimation of $D_{p}\left(\Omega_{i}\right)$}

Let $\Gamma$ be the gamma function, that is, $\Gamma(x)=\int_{0}^{\infty} t^{x-1} e^{-t} d t$ for $x>0$. For $f \in L^{r}\left(\mathbb{R}^{N}\right)$ and $g \in L^{s}\left(\mathbb{R}^{N}\right)(1 \leq r, s \leq \infty)$, let $f * g: \mathbb{R}^{N} \rightarrow \mathbb{R}$ be the convolution of $f$ and $g$ defined by

$$
(f * g)(x):=\int_{\mathbb{R}^{N}} f(x-y) g(y) d y\left(=\int_{\mathbb{R}^{N}} f(x) g(x-y) d y\right) .
$$

In the following three lemmas, we recall some known results required to obtain explicit values of $D_{p}\left(\Omega_{i}\right)$ in (5) for bounded convex domains $\Omega_{i}$.

Lemma 3.1 (see, e.g., [30,31]) Let $\Omega \subset \mathbb{R}^{N}(N \in \mathbb{N})$ be a bounded convex domain. For $u \in W^{1,1}(\Omega)$ and any point $x \in \Omega$, we have

$$
\left|u(x)-u_{\Omega}(x)\right| \leq \frac{d_{\Omega}^{N}}{N|\Omega|} \int_{\Omega}|x-y|^{1-N}|\nabla u(y)| d y .
$$

A proof of Lemma 3.1 is provided in Appendix 2 because Lemma 3.1 plays an especially important role in obtaining the explicit values of $D_{p}\left(\Omega_{i}\right)$.

Lemma 3.2 (Hardy-Littlewood-Sobolev's inequality [32]) For $\lambda>0$, we put $h_{\lambda}(x):=|x|^{-\lambda}$. If $0<\lambda<N$,

$$
\left\|h_{\lambda} * g\right\|_{L^{\frac{2 N}{\lambda}}\left(\mathbb{R}^{N}\right)} \leq C_{\lambda, N}\|g\|_{L^{\frac{2 N}{2 N-\lambda}}\left(\mathbb{R}^{N}\right)} \text { for all } g \in L^{\frac{2 N}{2 N-\lambda}}\left(\mathbb{R}^{N}\right)
$$


holds valid for

$$
C_{\lambda, N}=\pi^{\frac{\lambda}{2}} \frac{\Gamma\left(\frac{N}{2}-\frac{\lambda}{2}\right)}{\Gamma\left(N-\frac{\lambda}{2}\right)}\left(\frac{\Gamma\left(\frac{N}{2}\right)}{\Gamma(N)}\right)^{-1+\frac{\lambda}{N}}
$$

where this is the best constant in (8).

Moreover, if $N<2 \lambda<2 N$,

$$
\left\|h_{\lambda} * g\right\|_{L^{2 \lambda-N}\left(\mathbb{R}^{N}\right)} \leq \tilde{C}_{\lambda, N}\|g\|_{L^{2}\left(\mathbb{R}^{N}\right)} \text { for all } g \in L^{2}\left(\mathbb{R}^{N}\right)
$$

holds valid for

$$
\tilde{C}_{\lambda, N}=\pi^{\frac{\lambda}{2}} \frac{\Gamma\left(\frac{N}{2}-\frac{\lambda}{2}\right)}{\Gamma\left(\frac{\lambda}{2}\right)} \sqrt{\frac{\Gamma\left(\lambda-\frac{N}{2}\right)}{\Gamma\left(\frac{3 N}{2}-\lambda\right)}}\left(\frac{\Gamma\left(\frac{N}{2}\right)}{\Gamma(N)}\right)^{-1+\frac{\lambda}{N}},
$$

where this is the best constant in (10).

Lemma 3.3 (Young's inequality [33]) Suppose that $1 \leq t, r, s \leq \infty$ and $1 / t=1 / r+1 / s-1 \geq$ 0 . For $f \in L^{r}\left(\mathbb{R}^{N}\right)$ and $g \in L^{s}\left(\mathbb{R}^{N}\right)$, we have

$$
\|f * g\|_{L^{t}\left(\mathbb{R}^{N}\right)} \leq\left(A_{r} A_{s} A_{t^{\prime}}\right)^{N}\|f\|_{L^{r}\left(\mathbb{R}^{N}\right)}\|g\|_{L^{s}\left(\mathbb{R}^{N}\right)}
$$

with

$$
A_{m}= \begin{cases}\sqrt{m^{\frac{2}{m}-1}(m-1)^{1-\frac{1}{m}}} & (1<m<\infty), \\ 1 & (m=1, \infty) .\end{cases}
$$

The constant $\left(A_{r} A_{s} A_{t^{\prime}}\right)^{N}$ is the best constant in (12).

The following Theorems 3.1, 3.2, 3.3, and 3.4 provide estimations of $D_{p}(\Omega)$ for a bounded convex domain $\Omega$, where $p, q$, and $N$ are imposed on the assumptions listed in Table 1 .

Theorem 3.1 Let $\Omega \subset \mathbb{R}^{N}(N \in \mathbb{N})$ be a bounded convex domain. Assume that $p \in \mathbb{R}$ satisfies $2<p \leq 2 N /(N-1)$ if $N \geq 2$ and $2<p<\infty$ if $N=1$. For $q \in \mathbb{R}$ such that $q \geq p /(p-1)$, we have

$$
\left\|u-u_{\Omega}\right\|_{L^{p}(\Omega)} \leq D_{p}(\Omega)\|\nabla u\|_{L^{q}(\Omega)} \quad \text { for all } u \in W^{1, q}(\Omega)
$$

with

$$
D_{p}(\Omega)=\frac{d_{\Omega}^{1+\frac{2 N}{p}} \pi^{\frac{N}{p}}}{N|\Omega|^{\frac{1}{p}+\frac{1}{q}}} \frac{\Gamma\left(\frac{p-2}{2 p} N\right)}{\Gamma\left(\frac{p-1}{p} N\right)}\left(\frac{\Gamma(N)}{\Gamma\left(\frac{N}{2}\right)}\right)^{\frac{p-2}{p}} .
$$


Proof Let $u \in W^{1, q}(\Omega)$. Since $p \leq 2 N /(N-1)$ and $1-N+(2 N / p) \geq 0$, it follows that $|x-z|^{1-N+\frac{2 N}{p}} \leq d_{\Omega}^{1-N+\frac{2 N}{p}}$ for $x, z \in \Omega$. Lemma 3.1 implies that, for a fixed $x \in \Omega$,

$$
\begin{aligned}
\left|u(x)-u_{\Omega}(x)\right| & \leq \frac{d_{\Omega}^{N}}{N|\Omega|} \int_{\Omega}|x-z|^{1-N+\frac{2 N}{p}}|x-z|^{-\frac{2 N}{p}}|\nabla u(z)| d z \\
& \leq \frac{d_{\Omega}^{1+\frac{2 N}{p}}}{N|\Omega|} \int_{\Omega}|x-z|^{-\frac{2 N}{p}}|\nabla u(z)| d z \\
& \leq \frac{d_{\Omega}^{1+\frac{2 N}{p}}}{N|\Omega|} \int_{\mathbb{R}^{N}}|x-z|^{-\frac{2 N}{p}}\left(E_{\Omega, \mathbb{R}^{N}}|\nabla u|\right)(z) d z
\end{aligned}
$$

Therefore,

$$
\begin{aligned}
\left\|u-u_{\Omega}\right\|_{L^{p}(\Omega)} & \leq \frac{d_{\Omega}^{1+\frac{2 N}{p}}}{N|\Omega|}\left(\int_{\Omega}\left(\int_{\mathbb{R}^{N}}|x-z|^{-\frac{2 N}{p}}\left(E_{\Omega, \mathbb{R}^{N}}|\nabla u|\right)(z) d z\right)^{p} d x\right)^{\frac{1}{p}} \\
& \leq \frac{d_{\Omega}^{1+\frac{2 N}{p}}}{N|\Omega|}\left(\int_{\mathbb{R}^{N}}\left(\int_{\mathbb{R}^{N}}|x-z|^{-\frac{2 N}{p}}\left(E_{\Omega, \mathbb{R}^{N}}|\nabla u|\right)(z) d z\right)^{p} d x\right)^{\frac{1}{p}} .
\end{aligned}
$$

Since $q \geq p /(p-1)$ and $\Omega$ is bounded, we have $|\nabla u| \in L^{p /(p-1)}(\Omega)$. Therefore, Lemma 3.2 ensures

$$
\begin{aligned}
\left\|u-u_{\Omega}\right\|_{L^{p}(\Omega)} & \leq \frac{d_{\Omega}^{1+\frac{2 N}{p}}}{N|\Omega|} C_{\frac{2 N}{p}, N}\left\|E_{\Omega, \mathbb{R}^{N}}|\nabla u|\right\|_{L^{\frac{p}{p-1}}\left(\mathbb{R}^{N}\right)} \\
& =\frac{d_{\Omega}^{1+\frac{2 N}{p}}}{N|\Omega|} C_{\frac{2 N}{p}, N}\|\nabla u\|_{L^{\frac{p}{p-1}}(\Omega)}
\end{aligned}
$$

where $C_{\frac{2 N}{p}, N}$ is defined in (9) with $\lambda=2 N / p$. Since $q \geq p /(p-1)$, Hölder's inequality moreover implies

$$
\left\|u-u_{\Omega}\right\|_{L^{p}(\Omega)} \leq \frac{d_{\Omega}^{1+\frac{2 N}{p}}}{N|\Omega|^{\frac{1}{p}+\frac{1}{q}}} C_{\frac{2 N}{p}, N}\|\nabla u\|_{L^{q}(\Omega)} .
$$

Theorem 3.2 Let $\Omega \subset \mathbb{R}^{N}(N \geq 2)$ be a bounded convex domain. Assume that $2<p \leq$ $2 N /(N-2)$ if $N \geq 3$ and $2<p<\infty$ if $N=2$. For all $u \in W^{1,2}(\Omega)$, we have

$$
\left\|u-u_{\Omega}\right\|_{L^{p}(\Omega)} \leq D_{p}(\Omega)\|\nabla u\|_{L^{2}(\Omega)}
$$

with

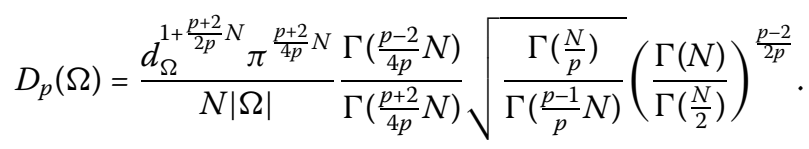


Proof Let $u \in W^{1,2}(\Omega)$. Since $p \leq 2 N /(N-2)$, it follows that $|x-z|^{1-N+(p+2) N /(2 p)} \leq$ $d_{\Omega}^{1-N+(p+2) N /(2 p)}$ for $x, z \in \Omega$. Lemma 3.1 leads to

$$
\begin{aligned}
\left|u(x)-u_{\Omega}(x)\right| & \leq \frac{d_{\Omega}^{N}}{N|\Omega|} \int_{\Omega}|x-z|^{1-N+\frac{p+2}{2 p} N}|x-z|^{-\frac{p+2}{2 p} N}|\nabla u(z)| d z \\
& \leq \frac{d_{\Omega}^{1+\frac{p+2}{2 p} N}}{N|\Omega|} \int_{\Omega}|x-z|^{-\frac{p+2}{2 p} N}|\nabla u(z)| d z \\
& \leq \frac{d_{\Omega}^{1+\frac{p+2}{2 p} N}}{N|\Omega|} \int_{\mathbb{R}^{N}}|x-z|^{-\frac{p+2}{2 p} N}\left(E_{\Omega, \mathbb{R}^{N}}|\nabla u|\right)(z) d z .
\end{aligned}
$$

Therefore,

$$
\begin{aligned}
\left\|u-u_{\Omega}\right\|_{L^{p}(\Omega)} & \leq \frac{d_{\Omega}^{1+\frac{p+2}{2 p} N}}{N|\Omega|}\left(\int_{\Omega}\left(\int_{\mathbb{R}^{N}}|x-z|^{-\frac{p+2}{2 p} N}\left(E_{\Omega, \mathbb{R}^{N}}|\nabla u|\right)(z) d z\right)^{p} d x\right)^{\frac{1}{p}} \\
& \leq \frac{d_{\Omega}^{1+\frac{p+2}{2 p} N}}{N|\Omega|}\left(\int_{\mathbb{R}^{N}}\left(\int_{\mathbb{R}^{N}}|x-z|^{-\frac{p+2}{2 p} N}\left(E_{\Omega, \mathbb{R}^{N}}|\nabla u|\right)(z) d z\right)^{p} d x\right)^{\frac{1}{p}}
\end{aligned}
$$

From (10), it follows that

$$
\begin{aligned}
\left\|u-u_{\Omega}\right\|_{L^{p}(\Omega)} & \leq \frac{d_{\Omega}^{1+\frac{p+2}{2 p} N}}{N|\Omega|} \tilde{C}_{\frac{p+2}{2 p} N, N}\left\|E_{\Omega, \mathbb{R}^{N}}|\nabla u|\right\|_{L^{2}\left(\mathbb{R}^{N}\right)} \\
& =\frac{d_{\Omega}^{1+\frac{p+2}{2 p} N}}{N|\Omega|} \tilde{C}_{\frac{p+2}{2 p} N, N}\|\nabla u\|_{L^{2}(\Omega)},
\end{aligned}
$$

where $\tilde{C}_{\frac{p+2}{2 p} N, N}$ is defined in (11) with $\lambda=(p+2) N /(2 p)$.

Theorem 3.3 Let $\Omega \subset \mathbb{R}^{N}(N \in \mathbb{N})$ be a bounded convex domain. Suppose that $1 \leq q \leq$ $p<q N /(N-q)$ if $N>q$, and $1 \leq q \leq p<\infty$ if $N=q$. Then we have

$$
\left\|u-u_{\Omega}\right\|_{L^{p}(\Omega)} \leq D_{p}(\Omega)\|\nabla u\|_{L^{q}(\Omega)} \quad \text { for all } u \in W^{1, q}(\Omega)
$$

with

$$
D_{p}(\Omega)=\frac{d_{\Omega}^{N}}{N|\Omega|}\left(A_{r} A_{q} A_{p^{\prime}}\right)^{N}\left\||x|^{1-N}\right\|_{L^{r}(V)},
$$

where $\Omega_{x}:=\{x-y \mid y \in \Omega\}$ for $x \in \Omega, V:=\bigcup_{x \in \Omega} \Omega_{x}$, and $r=q p /((q-1) p+q)$.

Proof First, we prove $I:=\left\||x|^{1-N}\right\|_{L^{r}(V)}^{r}<\infty$. Let $\rho=2 d_{\Omega}$ so that $V \subset B(0, \rho)$. We have

$$
\begin{aligned}
\frac{p q(1-N)}{(q-1) p+q}+N-1 & =\frac{p q(1-N)+N p(q-1)+N q}{(q-1) p+q}-1 \\
& =\frac{N q-(N-q) p}{(q-1) p+q}-1>-1
\end{aligned}
$$


Therefore,

$$
I=\int_{V}|x|^{\frac{p q(1-N)}{(q-1) p+q}} d x \leq \int_{B(0, \rho)}|x|^{\frac{p q(1-N)}{(q-1) p+q}} d x=J \int_{0}^{\rho} \rho^{\frac{p q(1-N)}{(q-1) p+q}+N-1} d \rho<\infty
$$

where $J$ is defined by

$$
J= \begin{cases}2 & (N=1) \\ 2 \pi & (N=2), \\ 2 \pi \int_{[0, \pi]^{N-2}} \prod_{i=1}^{N-2}\left(\sin \theta_{i}\right)^{N-i-1} d \theta_{1} \cdots d \theta_{N-2} & (N \geq 3) .\end{cases}
$$

Next, we show (13). For $x \in \Omega$, it follows from Lemma 3.1 that

$$
\begin{aligned}
\left|u(x)-u_{\Omega}(x)\right| & \leq \frac{d_{\Omega}^{N}}{N|\Omega|} \int_{\Omega}|x-y|^{1-N}|\nabla u(y)| d y \\
& =\frac{d_{\Omega}^{N}}{N|\Omega|} \int_{\Omega_{x}}|y|^{1-N}|\nabla u(x-y)| d y \\
& \leq \frac{d_{\Omega}^{N}}{N|\Omega|} \int_{V}|y|^{1-N}\left(E_{\Omega, V}|\nabla u|\right)(x-y) d y .
\end{aligned}
$$

Since $E_{V, \mathbb{R}^{N}} E_{\Omega, V}=E_{\Omega, \mathbb{R}^{N}}$,

$$
\left|u(x)-u_{\Omega}(x)\right| \leq \frac{d_{\Omega}^{N}}{N|\Omega|} \int_{\mathbb{R}^{N}}\left(E_{V, \mathbb{R}^{N}} \psi\right)(y)\left(E_{\Omega, \mathbb{R}^{N}}|\nabla u|\right)(x-y) d y,
$$

where $\psi(y)=|y|^{1-N}$ for $y \in V$. We denote $f(x)=\left(E_{V, \mathbb{R}^{N}} \psi\right)(x)$ and $g(x)=\left(E_{\Omega, \mathbb{R}^{N}}|\nabla u|\right)(x)$. Lemma 3.3 and (14) give

$$
\begin{aligned}
\left\|u-u_{\Omega}\right\|_{L^{p}(\Omega)} & \leq \frac{d_{\Omega}^{N}}{N|\Omega|}\|f * g\|_{L^{p}(\Omega)} \\
& \leq \frac{d_{\Omega}^{N}}{N|\Omega|}\|f * g\|_{L^{p}\left(\mathbb{R}^{N}\right)} \\
& \leq \frac{d_{\Omega}^{N}}{N|\Omega|}\left(A_{r} A_{q} A_{p^{\prime}}\right)^{N}\|f\|_{L^{r}\left(\mathbb{R}^{N}\right)}\|g\|_{L^{q}\left(\mathbb{R}^{N}\right)} \\
& =\frac{d_{\Omega}^{N}}{N|\Omega|}\left(A_{r} A_{q} A_{p^{\prime}}\right)^{N} I^{\frac{1}{r}}\|\nabla u\|_{L^{q}(\Omega)} .
\end{aligned}
$$

Theorem 3.4 Let $\Omega \subset \mathbb{R}^{N}(N \in \mathbb{N})$ be a bounded convex domain, and let $q>N$. Then we have

$$
\left\|u-u_{\Omega}\right\|_{L^{\infty}(\Omega)} \leq D_{\infty}(\Omega)\|\nabla u\|_{L^{q}(\Omega)} \quad \text { for all } u \in W^{1, q}(\Omega)
$$

with

$$
D_{\infty}(\Omega)=\frac{d_{\Omega}^{N}}{N|\Omega|}\left\||x|^{1-N}\right\|_{L^{q^{\prime}}(V)},
$$

where $V$ is defined in Theorem 3.3. 
Proof First, we show $I:=\left\||x|^{1-N}\right\|_{L^{q^{\prime}(V)}}^{q^{\prime}}<\infty$. Let $\rho=2 d_{\Omega}$ so that $V \subset B(0, \rho)$. We have

$$
q^{\prime}(1-N)+N-1=\frac{q(1-N)+N(q-1)}{q-1}-1=\frac{q-N}{q-1}-1>-1 .
$$

Therefore,

$$
I=\int_{V}|x|^{q^{\prime}(1-N)} d x \leq \int_{B(0, \rho)}|x|^{q^{\prime}(1-N)} d x=J \int_{0}^{\rho} \rho^{q^{\prime}(1-N)+N-1} d \rho<\infty
$$

where $J$ is defined in the proof of Theorem 3.3.

Next, we prove (15). Let $r=\frac{q}{q-1}(\geq 1), f(x)=\left(E_{V, \mathbb{R}^{N}} \psi\right)(x)$, and $g(x)=\left(E_{\Omega, \mathbb{R}^{N}}|\nabla u|\right)(x)$, where $\psi$ is denoted in the proof of Theorem 3.3. From Lemma 3.3 and (14), for $u \in$ $W^{1, q}(\Omega)$, it follows that

$$
\begin{aligned}
\left\|u-u_{\Omega}\right\|_{L^{\infty}(\Omega)} & \leq \frac{d_{\Omega}^{N}}{N|\Omega|}\|f * g\|_{L^{\infty}(\Omega)} \leq \frac{d_{\Omega}^{N}}{N|\Omega|}\|f * g\|_{L^{\infty}\left(\mathbb{R}^{N}\right)} \\
& \leq \frac{d_{\Omega}^{N}}{N|\Omega|}\|f\|_{L^{q^{\prime}}\left(\mathbb{R}^{N}\right)}\|g\|_{L^{q}\left(\mathbb{R}^{N}\right)}=\frac{d_{\Omega}^{N}}{N|\Omega|} I^{\frac{1}{q^{\prime}}}\|\nabla u\|_{L^{q}(\Omega)} .
\end{aligned}
$$

\section{Explicit values of $C_{p}(\Omega)$ for certain domains}

In this section, we present numerical examples where explicit values of $C_{p}(\Omega)$ on a square and a triangle domain are computed using Theorems 2.1, 3.1, 3.2, 3.3, and 3.4. All computations were performed on a computer with Intel Xeon E5-2687W @ $3.10 \mathrm{GHz}, 512$ GB RAM, CentOS 7, and MATLAB 2017a. All rounding errors were strictly estimated using the interval toolbox INTLAB version 10.1 [34]. Therefore, all values in the following tables are mathematically guaranteed to be upper bounds of the corresponding $C_{p}(\Omega)$ 's.

First, we select domains $\Omega_{i}(1 \leq i \leq n)$ satisfying (2) and (3). For all domains $\Omega_{i}(1 \leq$ $i \leq n)$, we then compute the values of $D_{p}\left(\Omega_{i}\right)$ using Theorems 3.1, 3.2, 3.3, and 3.4. Next, explicit values of $C_{p}(\Omega)$ are computed through Theorem 2.1.

\subsection{Estimation on a square domain}

For the first example, we select the case in which $\Omega=(0,1)^{2}$. For $n=1,4,16,64, \ldots$, we define each $\Omega_{i}(1 \leq i \leq n)$ as a square with side length $1 / \sqrt{n}$; see Figure 1 for the cases in
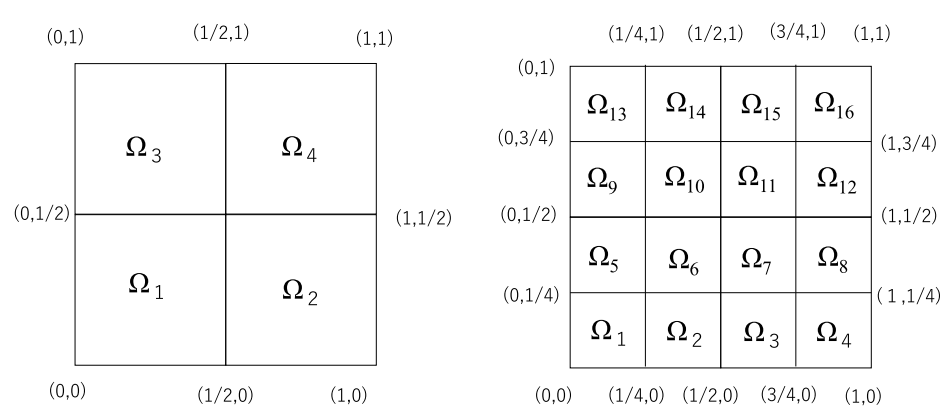

Figure $1 \Omega_{i}$ for the cases in which $n=4$ (the left-hand side) and $n=16$ (the right-hand side). 
Figure 2 The domain $V$ in Theorems 3.3 and 3.4.

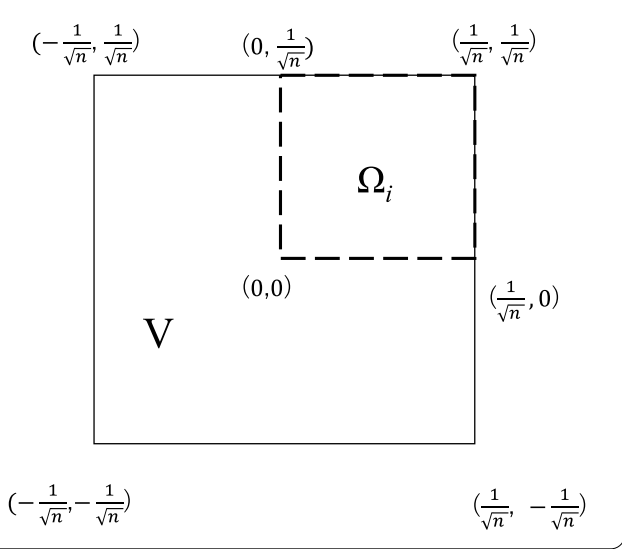

Table 2 Computed values of $C_{p}(\Omega)$ for $\Omega=(0,1)^{2}$ and $q=2$. The numbers of division $n$ are shown in the corresponding parentheses. Theorem 3.1 cannot be used for $p>4$ when $N=2$

\begin{tabular}{rlcccc}
\hline $\boldsymbol{p}$ & Theorem 3.1 & Theorem 3.2 & Theorem 3.3 & [10, Lemma 2.3] & [19, Corollary D.1] \\
\hline 3 & $2.553767(16)$ & $4.423506(256)$ & $2.647076(16)$ & 1.272533 & $1.291703 \times 10^{4}$ \\
4 & $2.506629(4)$ & $5.656855(256)$ & $3.098954(16)$ & 1.553774 & $1.809271 \times 10^{4}$ \\
5 & - & $5.721912(64)$ & $3.527578(16)$ & 1.841950 & $2.275458 \times 10^{4}$ \\
6 & - & $5.802230(64)$ & $3.922709(16)$ & 2.135792 & $2.701890 \times 10^{4}$ \\
7 & - & $6.245674(64)$ & $4.288114(16)$ & 2.434362 & $3.096661 \times 10^{4}$ \\
8 & - & $6.727172(64)$ & $4.628497(16)$ & 2.736941 & $3.465528 \times 10^{4}$ \\
9 & - & $7.127190(64)$ & $4.947849(16)$ & 3.042967 & $3.812726 \times 10^{4}$ \\
10 & - & $7.464264(64)$ & $5.249352(16)$ & 3.351991 & $4.141471 \times 10^{4}$ \\
& & & & \\
20 & - & $9.162396(16)$ & $7.659208(16)$ & 6.549949 & $6.789009 \times 10^{4}$ \\
30 & - & $10.202188(64)$ & $9.485455(16)$ & 9.856546 & $8.800592 \times 10^{4}$ \\
40 & - & $11.632217(64)$ & $10.640059(64)$ & 13.218367 & $1.048141 \times 10^{5}$ \\
50 & - & $12.907885(64)$ & $12.020066(64)$ & 16.613831 & $1.195208 \times 10^{5}$ \\
60 & - & $14.069728(64)$ & $13.258962(64)$ & 20.031993 & $1.327453 \times 10^{5}$ \\
70 & - & $15.143396(64)$ & $14.392550(64)$ & 23.466517 & $1.448540 \times 10^{5}$ \\
80 & - & $16.146231(64)$ & $15.443710(64)$ & 26.913400 & $1.560849 \times 10^{5}$ \\
\hline
\end{tabular}

which $n=4$ and $n=16$. For this division of $\Omega$, Theorem 2.1 states that

$$
C_{p}(\Omega)=2^{1-\frac{1}{q}} \max \left(n^{-\left(\frac{1}{p}-\frac{1}{q}\right)}, \max _{1 \leq i \leq n} D_{p}\left(\Omega_{i}\right)\right)
$$

In this case, $V$ (in Theorems 3.3 and 3.4) becomes a square with side length $2 / \sqrt{n}$ (see Figure 2). Note that $\left\||x|^{1-N}\right\|_{L^{r}(V)}=\int_{V}|x|^{\beta} d x$, where $\beta=q p(1-N) /((q-1) p+q)$ if $p<\infty$ and $\beta=q^{\prime}(1-N)$ if $p=\infty$.

Table 2 compares upper bounds for $C_{p}(\Omega)$ computed by Theorems 3.1, 3.2, 3.3, [10, Lemma 2.3], and [19, Corollary D.1] with $q=2$; the numbers of division $n$ are shown in the corresponding parentheses. Moreover, these values are plotted in Figure 3, except for the values derived from [19, Corollary D.1].

Theorems 3.1, 3.2, 3.3, and [10, Lemma 2.3] provide sharper estimates of $C_{p}(\Omega)$ than $[19$, Corollary D.1] for all $p$ 's. The estimates derived by Theorem 3.2 and Theorem 3.3 for $32 \leq p \leq 80$ are sharper than the estimates obtained by [10, Lemma 2.3].

We also show the values of $C_{\infty}(\Omega)$ computed by Theorem 3.4 for $3 \leq q \leq 10$ in Table 3 . 
Table 3 Computed values of $C_{\infty}(\Omega)$ for a square domain $\Omega$ and $3 \leq q \leq 10$. The numbers of division $n$ are shown in the corresponding parentheses

\begin{tabular}{rl}
\hline $\boldsymbol{q}$ & Theorem $\mathbf{3 . 4}$ \\
\hline 3 & $5.611920(16)$ \\
4 & $4.756829(64)$ \\
5 & $4.000001(64)$ \\
6 & $3.563595(64)$ \\
7 & $3.281342(64)$ \\
8 & $3.084422(64)$ \\
9 & $2.939469(64)$ \\
10 & $2.828428(64)$ \\
\hline
\end{tabular}

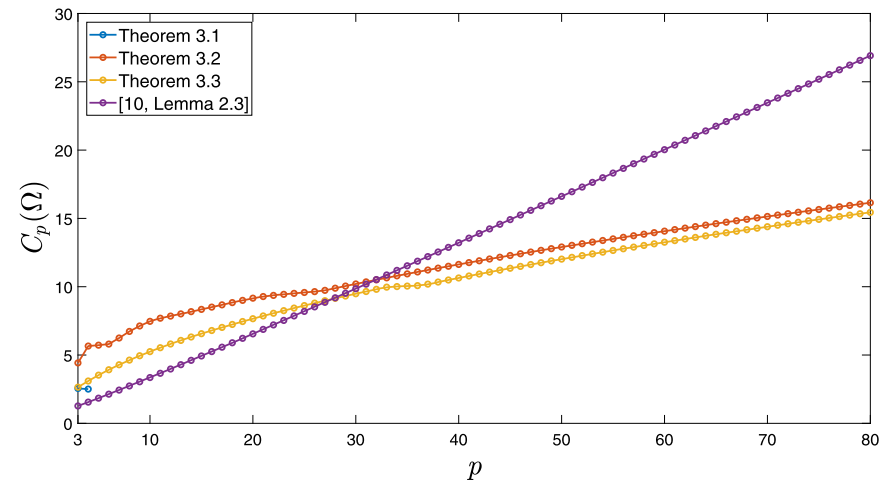

Figure 3 Computed values of $C_{p}(\Omega)$ for $\Omega=(0,1)^{2}$ and $3 \leq p \leq 80$.

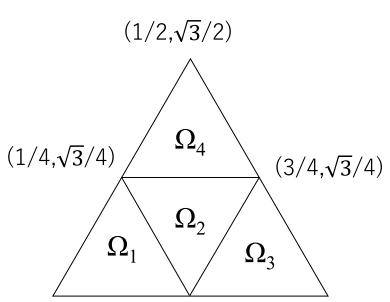

$(0,0)$

$(1 / 2,0)$

$(1,0)$

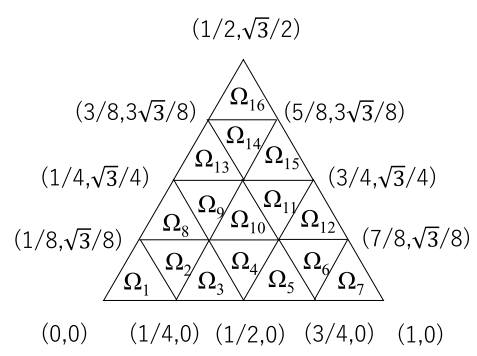

Figure $4 \Omega_{i}$ when $n=4$ (the left-hand side) and $n=16$ (the right-hand side).

\subsection{Estimation on a triangle domain}

For the second example, we select the case in which $\Omega$ is a regular triangle with the vertices $(0,0),(1,0)$, and $(1 / 2, \sqrt{3} / 2)$. For $n=1,4,16,64, \ldots$, we define each $\Omega_{i}(1 \leq i \leq n)$ as a regular triangle with side length $1 / \sqrt{n}$; see Figure 4 for the case in which $n=4$ and $n=16$. For this division of $\Omega$, Theorem 2.1 states that

$$
C_{p}(\Omega)=2^{1-\frac{1}{q}} \max \left(\left(\frac{4 n}{\sqrt{3}}\right)^{-\left(\frac{1}{p}-\frac{1}{q}\right)}, \max _{1 \leq i \leq n} D_{p}\left(\Omega_{i}\right)\right) .
$$

In this case, $V$ is the regular hexagon displayed in Figure 5.

Table 4 compares upper bounds of $C_{p}(\Omega)$ computed by Theorems 3.1, 3.2, 3.3, and [19, Corollary D.1] with $q=2$; the numbers of division $n$ are shown in the corresponding paren- 
Figure 5 The domain $V$ in Theorems 3.3 and 3.4.

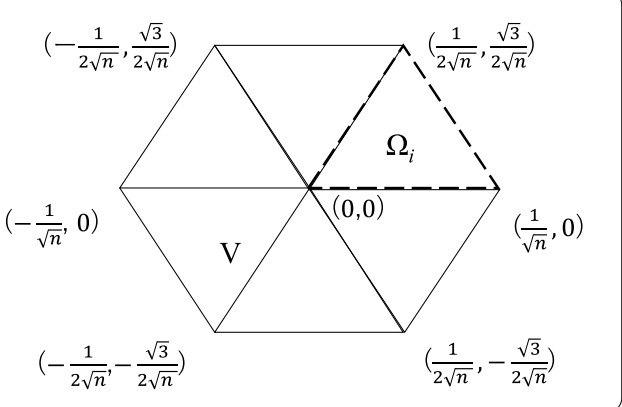

Table 4 Computed values of $C_{p}(\Omega)$ for a regular triangle domain $\Omega$ and $q=2$. The numbers of division $n$ are shown in the corresponding parentheses. Theorem 3.1 cannot be used for $p>4$ when $N=2$

\begin{tabular}{rlccc}
\hline $\boldsymbol{p}$ & Theorem 3.1 & Theorem 3.2 & Theorem 3.3 & [19, Corollary D.1] \\
\hline 3 & $2.580982(16)$ & $4.097053(256)$ & $2.366856(4)$ & $2.538335 \times 10^{4}$ \\
4 & $2.465500(4)$ & $5.700515(64)$ & $2.709475(4)$ & $3.553398 \times 10^{4}$ \\
5 & - & $6.330220(64)$ & $3.042818(4)$ & $4.464990 \times 10^{4}$ \\
6 & - & $7.477243(64)$ & $3.353176(4)$ & $5.297547 \times 10^{4}$ \\
7 & - & $7.601403(16)$ & $3.641844(4)$ & $6.067602 \times 10^{4}$ \\
8 & - & $7.750471(16)$ & $3.911816(4)$ & $6.786738 \times 10^{4}$ \\
9 & - & $7.933346(16)$ & $4.165864(4)$ & $7.463399 \times 10^{4}$ \\
10 & - & $8.133664(16)$ & $4.406282(4)$ & $8.103954 \times 10^{4}$ \\
& - & $10.219436(16)$ & $6.341217(4)$ & $1.326097 \times 10^{5}$ \\
30 & - & $12.055827(16)$ & $7.622031(16)$ & $1.717928 \times 10^{5}$ \\
40 & - & $13.666509(16)$ & $8.748299(16)$ & $2.045371 \times 10^{5}$ \\
50 & - & $15.112804(16)$ & $9.869218(16)$ & $2.331904 \times 10^{5}$ \\
60 & - & $16.059718(64)$ & $10.876336(16)$ & $2.589578 \times 10^{5}$ \\
70 & - & $17.313793(64)$ & $11.798394(16)$ & $2.825529 \times 10^{5}$ \\
80 & - & $18.483221(64)$ & $12.653794(16)$ & $3.044383 \times 10^{5}$ \\
\hline
\end{tabular}

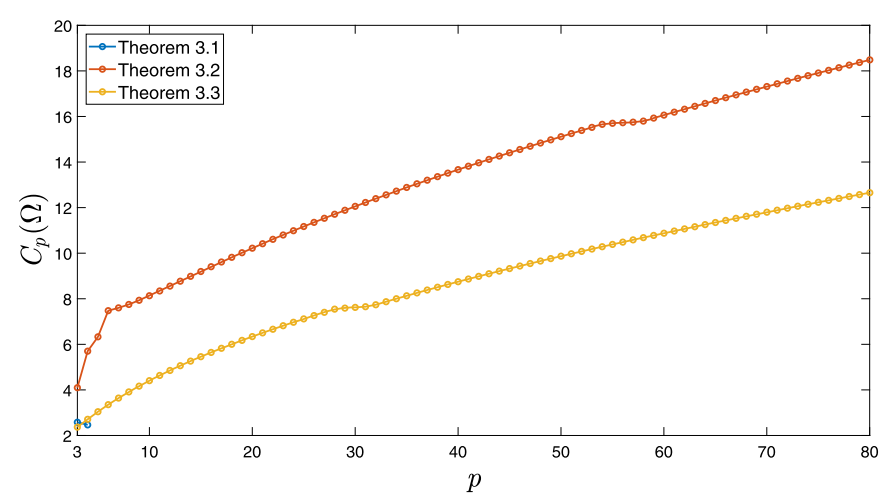

Figure 6 Computed values of $C_{p}(\Omega)$ for a regular triangle domain $\Omega$ and $3 \leq p \leq 80$.

theses. Moreover, these values are plotted in Figure 6 . The estimate computed by Theorem 3.1 is sharpest when $p=4$. However, for the other $p$ satisfying $3 \leq p \leq 80$, Theorem 3.3 provides the sharpest estimates.

We also show the values of $C_{\infty}(\Omega)$ computed by Theorem 3.4 for $3 \leq q \leq 10$ in Table 5 . 
Table 5 Computed values of $C_{\infty}(\Omega)$ for a regular triangle domain $\Omega$ and $3 \leq q \leq 10$. The numbers of division $n$ are shown in the corresponding parentheses

\begin{tabular}{rl}
\hline $\boldsymbol{q}$ & Theorem 3.4 \\
\hline 3 & $4.797133(4)$ \\
4 & $4.146459(16)$ \\
5 & $3.583834(16)$ \\
6 & $3.251833(16)$ \\
7 & $3.033691(16)$ \\
8 & $2.879743(16)$ \\
9 & $2.765427(16)$ \\
10 & $2.677251(16)$ \\
\hline
\end{tabular}

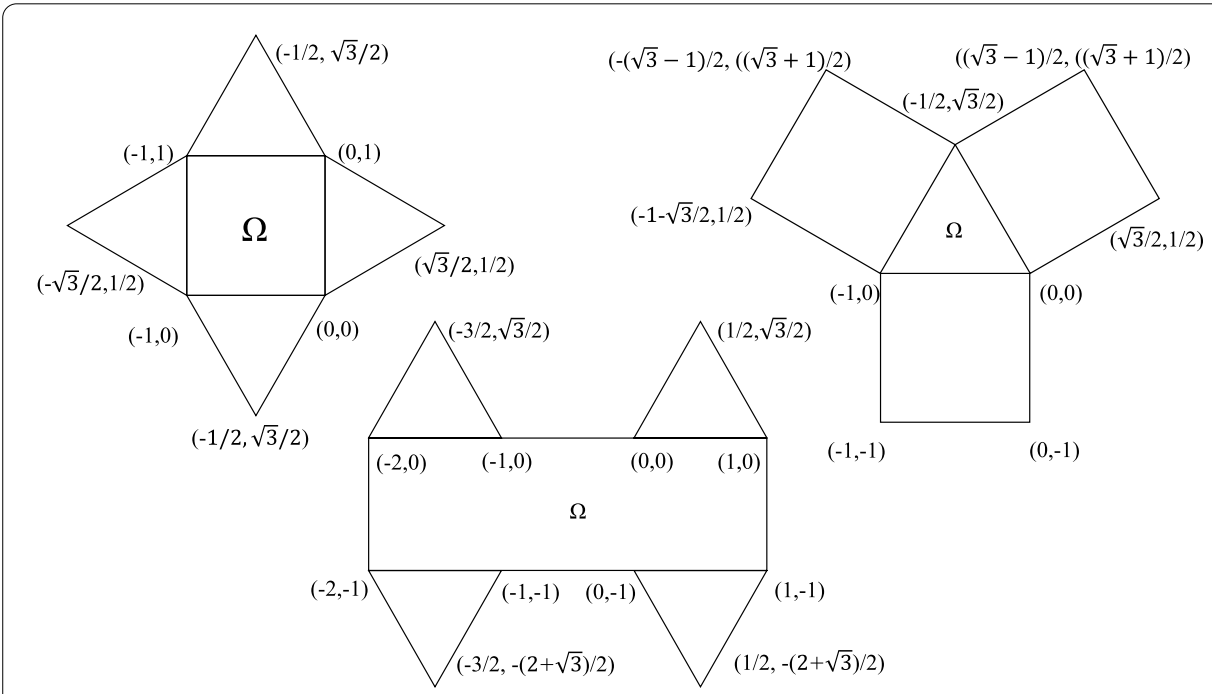

Figure 7 Some examples of domains $\Omega$ that are composed of unit squares and triangles with side length 1.

Remark 4.1 The values of $C_{p}(\Omega)$ derived from Theorem 3.1 to 3.4 (provided in Tables 1 to 5) can be directly used for any domain that is composed of unit squares and triangles with side length 1 (see Figure 7 for some examples).

\subsection{Estimation on a cube domain}

For the third example, we select the case in which $\Omega=(0,1)^{3}$. For $n=1,8,64,512, \ldots$, we define each $\Omega_{i}(1 \leq i \leq n)$ as a cube with side length $1 / \sqrt[3]{n}$. For this division of $\Omega$, Theorem 2.1 states that

$$
C_{p}(\Omega)=2^{1-\frac{1}{q}} \max \left(n^{-\left(\frac{1}{p}-\frac{1}{q}\right)}, \max _{1 \leq i \leq n} D_{p}\left(\Omega_{i}\right)\right)
$$

In this case, $V$ is also a cube with the side length $2 / \sqrt[3]{n}$.

Table 6 compares upper bounds of $C_{p}(\Omega)$ computed by Theorems 3.1, 3.2, 3.3, and [19, Corollary D.1] with $q=2$; the numbers of division $n$ are shown in the corresponding parentheses. The minimum value for each $p$ is written in bold. We also show the values of $C_{\infty}(\Omega)$ computed by Theorem 3.4 for $4 \leq q \leq 10$ in Table 7 . 
Table 6 Computed values of $C_{p}(\Omega)$ for a cube domain $\Omega$ and $q=2$. The numbers of division $n$ are shown in the corresponding parentheses. Theorem 3.1 for $p>3$ cannot be used when $N=3$. Theorem 3.2 can be used for $p=6$ only when $N=3$

\begin{tabular}{lllcl}
\hline $\boldsymbol{p}$ & Theorem 3.1 & Theorem 3.2 & Theorem 3.3 & [19, Corollary D.1] \\
\hline 3 & $\mathbf{4 . 0 0 0 0 0 1 ( 5 1 2 )}$ & $10.919242(32,768)$ & $5.947133(4096)$ & $3.115606 \times 10^{4}$ \\
4 & - & $16.340789(4096)$ & $\mathbf{1 3 . 2 4 1 2 4 5 ( 4 0 9 6 )}$ & $4.219101 \times 10^{4}$ \\
5 & - & $\mathbf{1 8 . 4 3 6 3 4 8 ( 4 0 9 6 )}$ & $29.676745(4096)$ & $5.239741 \times 10^{4}$ \\
6 & - & $\mathbf{2 0 . 6 5 8 4 7 1 ( 1 )}$ & - & - \\
\hline
\end{tabular}

Table 7 Computed values of $C_{\infty}(\Omega)$ for a cube domain $\Omega$ and $4 \leq q \leq 10$. The numbers of division $n$ are shown in the corresponding parentheses

\begin{tabular}{rr}
\hline $\boldsymbol{q}$ & Theorem 3.4 \\
\hline 4 & $22.627417(32,768)$ \\
5 & $13.928810(32,768)$ \\
6 & $10.079369(32,768)$ \\
7 & $8.000001(32,768)$ \\
8 & $6.727172(32,768)$ \\
9 & $5.878938(32,768)$ \\
10 & $5.278032(32,768)$ \\
\hline
\end{tabular}

\section{Conclusion}

We proposed several theorems that provide explicit values of Sobolev type embedding constant $C_{p}(\Omega)$ satisfying (1) for a domain $\Omega$ that can be divided into a finite number of bounded convex domains. These theorems give sharper estimates of $C_{p}(\Omega)$ than the previous estimates derived by the method in [19]. This accuracy improvement leads to much applicability of the estimates of $C_{p}(\Omega)$ to verified numerical computations for PDEs.

\section{Appendix 1: Embedding constant $C_{p}(\Omega)$ on dividable domains}

Theorem 6.1 provides an estimation of the embedding constant $C_{p}(\Omega)$ for a domain $\Omega$ that can be divided into domains $\Omega_{i}$ (such as convex domains and Lipschitz domains) satisfying (2) and (3).

Theorem 6.1 Let $\Omega \subset \mathbb{R}^{N}(N \in \mathbb{N})$ be a domain that can be divided into a finite number of domains $\Omega_{i}(i=1,2,3, \ldots, n)$ satisfying (2) and (3). Assume that, for every $\Omega_{i}(i=$ $1,2,3, \ldots, n)$, there exists a constant $C_{p}\left(\Omega_{i}\right)$ such that $\|u\|_{L^{p}\left(\Omega_{i}\right)} \leq C_{p}\left(\Omega_{i}\right)\|u\|_{W^{1, q}\left(\Omega_{i}\right)}$ for all $u \in W^{1, q}\left(\Omega_{i}\right)$. Then (1) holds valid for

$$
C_{p}(\Omega)=M_{p, q} \max _{1 \leq i \leq n} C_{p}\left(\Omega_{i}\right)
$$

where

$$
M_{p, q}= \begin{cases}1 & (p \geq q) \\ n^{\frac{1}{p}-\frac{1}{q}} & (p<q) .\end{cases}
$$

Proof We consider both the cases in which $p<\infty$ and $p=\infty$. 
When $p<\infty$, it follows that

$$
\begin{aligned}
\|u\|_{L^{p}(\Omega)} & =\left(\sum_{1 \leq i \leq n}\|u\|_{L^{p}\left(\Omega_{i}\right)}^{p}\right)^{1 / p} \\
& \leq\left(\sum_{1 \leq i \leq n} C_{p}\left(\Omega_{i}\right)^{p}\|u\|_{W^{1, q}\left(\Omega_{i}\right)}^{p}\right)^{1 / p} \\
& \leq \max _{1 \leq i \leq n} C_{p}\left(\Omega_{i}\right)\left(\sum_{1 \leq i \leq n}\|u\|_{W^{1, q}\left(\Omega_{i}\right)}^{p}\right)^{1 / p} \\
& \leq M_{p, q} \max _{1 \leq i \leq n} C_{p}\left(\Omega_{i}\right)\|u\|_{W^{1, q}(\Omega)} .
\end{aligned}
$$

Note that $|x|_{p} \leq M_{p, q}|x|_{q}$ holds for $x=\left(x_{1}, x_{2}, \ldots, x_{n}\right) \in \mathbb{R}^{n}$ (see [19, Lemma A.1] for a detailed proof), where we denote

$$
|x|_{p}= \begin{cases}\left(\sum_{1 \leq i \leq n}\left|x_{i}\right|^{p}\right)^{\frac{1}{p}} & (1 \leq p<\infty), \\ \max _{1 \leq i \leq n}\left|x_{i}\right| & (p=\infty) .\end{cases}
$$

When $p=\infty$,

$$
\begin{aligned}
\|u\|_{L^{\infty}(\Omega)} & =\max _{1 \leq i \leq n}\|u\|_{L^{\infty}\left(\Omega_{i}\right)} \\
& \leq \max _{1 \leq i \leq n} C_{p}\left(\Omega_{i}\right)\|u\|_{W^{1, q}\left(\Omega_{i}\right)} \\
& \leq \max _{1 \leq i \leq n} C_{p}\left(\Omega_{i}\right) \max _{1 \leq i \leq n}\|u\|_{W^{1, q}\left(\Omega_{i}\right)} .
\end{aligned}
$$

Since $M_{\infty, q}=1$, we have

$$
\|u\|_{L^{\infty}(\Omega)} \leq \max _{1 \leq i \leq n} C_{p}\left(\Omega_{i}\right)\|u\|_{W^{1, q}(\Omega)} .
$$

\section{Appendix 2: A proof of Lemma 3.1}

This section provides a proof of Lemma 3.1 based on [31, Lemma 7.16].

Proof of Lemma 3.1 Since $C^{\infty}(\Omega) \cap W^{1,1}(\Omega)$ is densely defined in $W^{1,1}(\Omega)$, it suffices to prove Lemma 3.1 for $u \in C^{1}(\Omega)$. Since $\Omega$ is convex, we have, for $x, y \in \Omega$,

$$
u(x)-u(y)=-\int_{0}^{|x-y|} \partial_{r} u(x+r \omega) d r
$$

where $\omega=(y-x) /|y-x|$ and $\partial_{r} u(x+r \omega)=\frac{\partial}{\partial r} u(x+r \omega)$. Integrating with respect to $y$ over $\Omega$, we obtain

$$
\begin{aligned}
\left|u(x)-u_{\Omega}(x)\right| & =|\Omega|^{-1}\left|\int_{\Omega} \int_{0}^{|x-y|} \partial_{r} u(x+r \omega) d r d y\right| \\
& \leq|\Omega|^{-1} \int_{\Omega} \int_{0}^{|x-y|}\left|\partial_{r} u(x+r \omega)\right| d r d y
\end{aligned}
$$




$$
\begin{aligned}
& \leq|\Omega|^{-1} \int_{\Omega} \int_{0}^{\infty}\left|\left(E_{\Omega, \mathbb{R}^{N}} \partial_{r} u\right)(x+r \omega)\right| d r d y \\
& \leq|\Omega|^{-1} \int_{B\left(x, d_{\Omega}\right)} \int_{0}^{\infty}\left|\left(E_{\Omega, \mathbb{R}^{N}} \partial_{r} u\right)(x+r \omega)\right| d r d y \\
& =|\Omega|^{-1} \int_{0}^{d_{\Omega}} \int_{|\omega|=1} \int_{0}^{\infty}\left|\left(E_{\Omega, \mathbb{R}^{N}} \partial_{r} u\right)(x+r \omega)\right| \rho^{N-1} d r d \omega d \rho \\
& =|\Omega|^{-1} \int_{0}^{\infty} \int_{|\omega|=1} \int_{0}^{d_{\Omega}}\left|\left(E_{\Omega, \mathbb{R}^{N}} \partial_{r} u\right)(x+r \omega)\right| \rho^{N-1} d \rho d \omega d r \\
& =\frac{d_{\Omega}^{N}}{N|\Omega|} \int_{0}^{\infty} \int_{|\omega|=1}\left|\left(E_{\Omega, \mathbb{R}^{N}} \partial_{r} u\right)(x+r \omega)\right| d \omega d r \\
& =\frac{d_{\Omega}^{N}}{N|\Omega|} \int_{|\omega|=1} \int_{0}^{\infty}\left|\left(E_{\Omega, \mathbb{R}^{N}} \partial_{r} u\right)(x+r \omega)\right| r^{1-N} r^{N-1} d r d \omega \\
& =\frac{d_{\Omega}^{N}}{N|\Omega|} \int_{\mathbb{R}^{N}}\left|\left(E_{\Omega, \mathbb{R}^{N}} \partial_{r} u\right)(y)\right||x-y|^{1-N} d y \\
& =\frac{d_{\Omega}^{N}}{N|\Omega|} \int_{\Omega}\left|\partial_{r} u(y)\right||x-y|^{1-N} d y .
\end{aligned}
$$

Therefore, a proof of Lemma 3.1 is completed.

\section{Acknowledgements}

This work was supported by CREST, Japan Science and Technology Agency. The second author (KT) was supported by JSPS Grant-in-Aid for Research Activity Start-up Grant Number JP17H07188 and Mizuho Foundation for the Promotion of Sciences. The third author (KS) was supported by JSPS KAKENHI Grant Number 16K17651. We thank the editors and reviewers for giving useful comments to improve the contents of this manuscript.

\section{Competing interests}

The authors declare that they have no competing interests.

\section{Authors' contributions}

All authors contributed equally and significantly in writing this paper. All authors read and approved the final manuscript.

\section{Author details}

${ }^{1}$ Faculty of Science and Engineering, Waseda University, 3-4-1, Okubo Shinjyuku-ku, Tokyo, 169-8555, Japan. ${ }^{2}$ Faculty of Information Networking for Innovation and Design, Toyo University, 1-7-11 Akabanedai, kita-ku, Tokyo, 115-0053, Japan.

\section{Publisher's Note}

Springer Nature remains neutral with regard to jurisdictional claims in published maps and institutional affiliations.

Received: 6 July 2017 Accepted: 17 November 2017 Published online: 29 November 2017

\section{References}

1. Sobolev, SL: On a theorem of functional analysis. Mat. Sb. 4, 471-497 (1938)

2. Adams, RA: Sobolev Spaces, 2nd edn. Academic Press, New York (1975)

3. Whitney, H: Analytic extensions of differentiable functions defined in closed set. Trans. Am. Math. Soc. 36, 63-89 (1934)

4. Hestenes, MR: Extension of the range of a differential function. Duke Math J. 8, 183-192 (1941)

5. Calderón, AP: Lebesgue spaces of differentiable functions and distributions. In: Proc. Symp. Pure Math., vol. 4, pp. 33-49 (1961)

6. Stein, E: Singular Integrals and Differentiability Properties of Functions. Princeton University Press, Princeton (1970)

7. Roger, LG: Degree-independent Sobolev extension on locally uniform domains. J. Funct. Anal. 235, 619-665 (2006)

8. Nakao, MT: Numerical verification methods for solutions of ordinary and partial differential equations. Numer. Funct. Anal. Optim. 22, 321-356 (2001)

9. Plum, M: Existence and multiplicity proofs for semilinear elliptic boundary value problems by computer assistance. Jahresber. Dtsch. Math.-Ver. 110, 19-54 (2008)

10. Cai, S, Nagatou, K, Watanabe, Y: A numerical verification method a system of FitzHugh-Nagumo type. Numer. Funct. Anal. Optim. 33, 1195-1220 (2012)

11. Aubin, T: Probléms isopérimétriques et espaces de Sobolev. J. Differ. Geom. 11, 573-598 (1976)

12. Talenti, G: Best constant in Sobolev inequality. Ann. Math. Pures Appl. 110, 353-372 (1976) 
13. Kametaka, Y, Oshime, Y, Watanabe, $K$, Yamagishi, H, Nagai, A, Takemura, $K$ : The best constant of $L^{p}$ Sobolev inequality corresponding to the periodic boundary value problem for $(-1)^{M}(d / d x)^{2 M}$. Sci. Math. Jpn. 66, 169-181 (2007)

14. Oshime, $Y$, Kametaka, $Y$, Yamagishi, $H$ : The best constant of $L^{p}$ Sobolev inequality corresponding to the Dirichlet boundary value problem for $(d / d x)^{4 m}$. Sci. Math. Jpn. 68, 461-469 (2008)

15. Oshime, $Y$, Watanabe, $K$ : The best constant of $L^{P}$ Sobolev inequality corresponding to the Dirichlet boundary value problem ii. Tokyo J. Math. 34, 115-133 (2011)

16. Oshime, $Y$, Yamagishi, $H$, Watanabe, $K$ : The best constant of $L^{p}$ Sobolev inequality corresponding to Neumann boundary value problem for $(-1)^{M}(d / d x)^{2 M}$. Hiroshima Math. J. 42, 293-299 (2012)

17. Watanabe, K, Kametaka, Y, Yamagishi, H, Nagai, A, Takemura, K: The best constant of Sobolev inequality corresponding to clamped boundary value problem. Bound. Value Probl. 2011, 875057 (2011)

18. Tanaka, K, Sekine, K, Mizuguchi, M, Oishi, S: Sharp numerical inclusion of the best constant for embedding $H_{0}^{1}(\Omega) \hookrightarrow L^{p}(\Omega)$ on bounded domain. J. Comput. Appl. Math. 311, 306-313 (2017)

19. Tanaka, K, Sekine, K, Mizuguchi, M, Oishi, S: Estimation of Sobolev-type embedding constant on domains with minimally smooth boundary using extension operator. J. Inequal. Appl. 2015, 389 (2015)

20. Grisvard, P: Elliptic Problems in Nonsmooth Domains. SIAM, Philadelphia (2011)

21. Koskela, P, Onninen, J, Tyson, JT: Quasihyperbolic boundary conditions and Poincaré domains. Math. Ann. 323, 811-830 (2002)

22. Hajłasz, P, Koskela, P: Sobolev Met Poincaré. Mem. Amer. Math. Soc., vol. 145 (2000)

23. Bojarski, B: Remarks on Sobolev imbedding inequalities. In: Laine, I, Sorvali, T, Rickman, S (eds.) Complex Analysis Joensuu 1987. Lecture Notes in Mathematics, vol. 1351, pp. 52-68. Springer, Berlin (1988)

24. Nazarov, Al, Poborchi, SV: On the conditions of validity for the Poincaré inequality. Zap. Nauč. Semin. POMI 410 104-109 (2013)

25. Bennewitz, C, Saitō, Y: Approximation numbers of Sobolev embedding operators on an interval. J. Lond. Math. Soc. 70, 244-260 (2004)

26. Lu, G, Wheeden, RL: Poincaré inequalities, isoperimetric estimates and representation formulas on product spaces. Indiana Univ. Math. J. 47, 123-152 (1988)

27. Chua, S-K, Wheeden, RL: Sharp conditions for weighted 1-dimensional Poincaré inequalities. Indiana Univ. Math. J. 49 $143-175(2000)$

28. Acosta, G, Durán, RG: An optimal Poincaré inequality in L' for convex domains, Proc. Am. Math. Soc., 132, 195-202 (2004)

29. Payne, LE, Weinberger, HF: An optimal Poincaré inequality for convex domains. Arch. Ration. Mech. Anal. 5, 286-292 (1960)

30. Davies, EB: Heat Kernels and Spectral Theory. Cambridge University Press, New York (1989)

31. Gilbarg, D, Trudinger, NS: Elliptic Partial Differential Equations of Second Order. Springer, Berlin (1977)

32. Lieb, EH: Sharp constants in the Hardy-Littlewood-Sobolev and related inequalities. Ann. Math. 118, 349-374 (1983)

33. Becker, W: Inequalities in Fourier analysis. Ann. Math. 102, 159-182 (1975)

34. Rump, SM: Intlab - interval laboratory. In: Developments in Reliable Computing, pp. 77-104. Kluwer Academic, Dordrecht (1999)

\section{Submit your manuscript to a SpringerOpen ${ }^{\circ}$ journal and benefit from:}

- Convenient online submission

- Rigorous peer review

- Open access: articles freely available online

- High visibility within the field

- Retaining the copyright to your article

Submit your next manuscript at $\boldsymbol{s p r i n g e r o p e n . c o m ~}$ 